\title{
EFECTO DE CUATRO NIVELES PROTÉICOS PROVENIENTES DE LA HARINA DE SACHA INCHI Plukenetia volubilis (EUPHORBIACEAE) EN EL CRECIMIENTO DE ALEVINOS DE BANDA NEGRA Myleus schomburgkii (PISCES, SERRASALMIDAE) CRIADOS EN CAUTIVERIO
}

\author{
James GARCIA-AYALA ${ }^{1}$, Julio VILLA-LAVY², Luis MORI-PINEDO² \\ 1 Laboratorio de Biología e Ecología de Peixes - IBB -UNESP. Botucatu, SP. Brasil. jamesrga@hotmail.com. \\ 2 Universidad Nacional de la Amazonia Peruana - UNAP. Facultad de Ciencia Biológicas - UNAP.Iquitos, Perú.
}

\begin{abstract}
RESUMEN
En el presente estudio tuvo como objetivo evaluar el crecimiento de alevinos de banda negra Myleus schomburgkii, utilizando cuatro niveles de proteína bruta $(23,25,27$ y $29 \%)$ provenientes de la harina de sacha inchi Plukenetia volubilis (Euphorbiaceae). Para lo cual se utilizaron un total de 48 ejemplares de M.schomburgkii, los cuales fueron distribuidos en 12 jaulas flotantes con una densidad de siembra de cuatro ind./jaula. Los animales fueron alimentados diariamente durante 168 días, con una tasa de alimentación equivalente al $4 \%$ de la biomasa presente en cada jaula. Los peces de las dietas T1, T3 y T4 alcanzaron un peso promedio de $50.67 ; 51.50$ y $51.42 \mathrm{~g}$. los resultados muestran que los peces alimentados con la dieta T2 tuvieron un crecimiento superior obteniendo al final del experimento un peso promedio de $60.67 \mathrm{~g}$. Sin embargo todas las variables analizadas como peso final, tasa de crecimiento relativo y tasa de crecimiento especifico, No fueron influenciados significativamente $(\mathrm{P}>0.05)$ por ninguna de las dietas provenientes del sacha inchi. En términos de conversión alimenticia, los peces no fueron eficientes asimilando las dietas que les fueron ofertadas. Lo que indica que el desempeño productivo de los peces alimentados con dietas con elevado porcentaje de sacha inchi, Plukenetia volubilis en ración alimenticia para banda negra Myleus schomburgkii, no influyo significativamente $(\mathrm{P}>0.05)$ en su crecimiento tanto en peso como en longitud.
\end{abstract}

PALABRAS CLAVE: Myleus schomburgkii, Plukenetia volubilis, niveles de proteína, crecimiento, Amazonía peruana.

\section{THE EFFECT OF FOUR PROTEIN LEVELS OF THE SACHA INCHI FLOUR Plukenetia volubilis (EUPHORBIACEAE) IN THE GROWTH OF BLACK STRIPE YOUNG FISH Myleus schomburgkii (PISCES, SERRASALMIDAE) BREADED IN CAPTIVITY}

\begin{abstract}
The present study aimed to evaluate the growth of fingerlings of Myleus schomburgkii, using four levels of crude protein $(23,25,27$ and 29\%) from sacha inchi flour Plukenetia volubilis (Euphorbiaceae). Where was used a total of 48 specimens which were divided into $12 \mathrm{M}$. schomburgkii floating cages with a seeding density of four individuals per cage. The animals were fed daily for 168 days with a feed rate equivalent to $4 \%$ of the biomass in each cage. The fish diets T1, T3 and T4 reached an average weight of 50.67, 51.50 and 51.42 g. The results show that fish fed diet T2 had higher growth at the end of the experiment obtaining an average weight of $60.67 \mathrm{~g}$. However, all the variables analyzed as final weight, relative growth rate and specific growth rate, were not significantly influenced $(\mathrm{P}>0.05)$ by any of the diets from the sacha inchi. In terms of feed conversion the fish were not efficient assimilating the diet that was offered to them. This indicates that the productive performance of fish fed diets with high percentage of sacha inchi, Plukenetia volubilis in food ration for black band, schomburgkii Myleus was not significantly influenced $(\mathrm{P}>0.05)$ in growth in both weight and length.
\end{abstract}

KEYWORDS: Myleus schomburgkii, Plukenetia volubilis, levels of protein, growth, Peruvian Amazon. 


\section{INTRODUCCIÓN}

La piscicultura, constituye una alternativa comercial de producción de pescado capaz de atenuar y disminuir la presión de pesca sobre la fauna ictica, en especial de los peces de mayor valor comercial como gamitana Colossoma macropomum y paco Piaractus brachypomus que muestran signos de sobreexplotación (Guerra et al., 1996). La Amazonía Peruana posee condiciones adecuadas para el desarrollo de los cultivos acuícolas, orientados hacia una actividad económica con posibilidades de contribuir al PBI regional y nacional. El impacto económico más importante es la contribución a mejorar el nivel de vida de la población. En tal razón, la piscicultura está llamada a jugar un rol importante en esta región ya que además de bajar la presión de pesca, sobre los recursos, significa una oportunidad de generar puestos de trabajo, así como de crear un ambiente para la recreación y para la pesca comercial (Alcántara, 1990).

El potencial de crecimiento de la piscicultura está basado principalmente en la disponibilidad de agua, en la topología del área y en la habilidad para explorar nuevas especies que puedan ser cultivadas de una manera sostenida.

En la última década las discusiones sobre el impacto de la creciente introducción de especies exóticas ha generado la preocupación de los organismos conservacionistas, quienes vienen promoviendo el desarrollo de tecnologías para el cultivo de especies nativas promisorias que reemplacen a las especies exóticas o para ampliar el número de especies comúnmente cultivadas (Sánchez et al., 2005).

En este sentido banda negra, Myleus schomburgkii (Jardine, 1841), es una especie amazónica nativa, con hábitos migratorios relacionados con la disponibilidad de alimento (macrófitas y zooplancton) y periodos reproductivos, los cuales juegan un rol importante en el desarrollo biológico de la especie. Estos aspectos tienen que ser tomados en cuenta cuando la especie es criada en cautiverio, es decir tomar en consideración su hábito alimenticio, ritmo de crecimiento, condiciones físico-químicas en el cual se desarrolla la actividad, etc., para un mejor rendimiento de la especie (SaintPaul, 1986).

Otro de los limitantes para un mayor desarrollo de la piscicultura son los costos de producción, lo cual se elevan notoriamente, por el costo de alimentación de los peces durante el periodo de cultivo, existiendo la necesidad de buscar insumos locales. Intentando bajar estos costos es que actualmente se vienen desarrollando investigaciones en busca de alimentos alternativos tanto de origen vegetal como los de origen animal que puedan reemplazar a los usados tradicionalmente en dietas para peces.
En la Amazonía existen, muchos productos o subproductos que podrían ser utilizados como insumos en la formulación de raciones para los peces cultivados. Dentro de los productos de origen vegetal se encuentra el sacha inchi Plukenetia volubilis, una especie de la familia Euphorbiaceae que actualmente viene siendo también utilizada en la alimentación humana (Corazón-Guivin et al., 2008; 2009). Esta especie nativa de la Amazonía peruana, produce almendras que posee uno de los más altos contenidos de ácidos grasos esenciales para el organismo (omega 3, 6, y 9), diversos compuestos antioxidantes, además de poseer un elevado contenido proteico (29\%) de muy buena digestibilidad (Manco, 2006). En este sentido el presente trabajo pretende proporcionar información del crecimiento de alevinos de banda negra Myleus schomburgkii alimentados con cuatro dietas balanceadas, dentro de las cuales la harina de sacha inchi Plukenetia Volubilis, mesclados con otros subproductos contiene en cada tratamiento un elevado nivel proteico.

\section{MATERIALES Y MÉTODOS}

El trabajo fue realizado en el Centro de Investigación, Experimentación y EnseñanzaPiscigranja Quistococha-UNAP. Iquitos - Perú. Localizado en el Km 6 de la carretera Iquitos-Nauta, en el Caserío de Quistococha, Distrito de San Juan Bautista (Provincia de Maynas, del departamento de Loreto). Se utilizó un total de 48 peces de la especie Myleus schomburgkii de $26 \mathrm{~g}$ de peso y $10 \mathrm{~cm}$ de longitud inicial respectivamente, provenientes de la cuenca del río Nanay.

\section{FASE EXPERIMENTAL}

Fueron evaluados los efectos de cuatro niveles proteicos provenientes de la harina de sacha inchi plukenetia volubilis en el crecimiento de alevinos de banda negra Myleus schomburgkii criadas en cautiverio durante un periodo de 168 días. Para lo cual se utilizaron 12 jaulas flotantes de $1 \times 1 \times 1 \mathrm{~m}^{3}$, construidas con un armazón de tubos de PVC de 1" cubiertas por mallas de plástico de $2 \mathrm{~mm}$. De abertura, estas jaulas estaba sumergidas en un estanque de tierra de aproximadamente $517.5 \mathrm{~m}^{2}$ de espejo de agua.

En la experimentación fueron empleados 48 especímenes de banda negra, con una densidad de siembra de cuatro individuos por jaula. El diseño experimental consistió en cuatros dietas $(\mathrm{T} 1=23 \%$, $\mathrm{T} 2=25 \%, \mathrm{~T} 3=27 \%$ y $\mathrm{T} 4=29 \%$ de proteína bruta), con tres repeticiones cada una, con los siguientes 
ingredientes: harina de pescado, harina de sacha inchi, polvillo de arroz y harina de maíz. La harina de pescado es la única fuente de proteína animal la cual se encuentra en proporciones iguales $(10 \%)$ para que no tenga influencia en los resultados. Cabe indicar que todas las dietas experimentales tuvieron un elevado porcentaje de nivel de proteína proveniente de la harina de sacha inchi $(45,55,65,75 \%)$ por el método de regresión polinomial. Las raciones fueron realizadas de manera manual usando una maquina peletizadora con dados de criba de $4 \mathrm{~mm}$ de diámetro. Para la designación de los diferentes tenores proteicos por jaula se utilizó un diseño completamente al azar, con la finalidad de evitar el efecto de borde. La composición nutricional de las cuatro dietas utilizadas en el experimento se muestra en la tabla 1.

Los peces fueron alimentados los tres primeros meses, tres veces al día (7:30 AM; 12:30 PM y 5:30 PM), y los siguientes tres meses, fueron alimentados 2 veces al día (8:00AM y 5:00PM). En ambos periodos se administró alimento a razón del 4\% de la biomasa de cada jaula.

\section{MONITOREO DE LOS FACTORES FÍSICO-QUÍMICOS DEL AGUA}

Fue realizado tanto fuera como dentro de las jaulas a través de mediciones mensuales de temperatura y oxígeno, utilizando el Oxímetro YSI Modelo 55, mientras que las mediciones de $\mathrm{pH}$, Alcalinidad, Dióxido de Carbono, Dureza, Amoniaco, Nitritos y Nitratos, se hicieron con la ayuda de un Kit de Reactivos AQ-2 LaMotte.

\section{EVALUACIONES BIOMÉTRICAS E INDICES ZOOTECNICOS}

Fueron verificados el peso (g) y la longitud estándar (cm) de los especímenes en intervalos de 28 días sobre el $100 \%$ de la población presente en cada jaula. Los cuales tuvieron un peso promedio de $26 \mathrm{~g}$ y una longitud promedio de $10 \mathrm{~cm}$.

Los índices zootécnicos considerados fueron: Peso final (PF), Longitud final (LF), Tasa de crecimiento Relativo (LF), Tasa de crecimiento Relativo (TCR), Tasa de Crecimiento Especifico (TCR), Factor de Condición (K), Tasa de sobrevivencia, y el Índice de Conversión Alimenticia Aparente (ICAA). Para el cálculo de los ICAA, se consideró un reajuste a la cantidad total de alimento consumido por los peces de cada unidad experimental.

\section{ANALISIS BROMATOLOGICO}

Los análisis del ingrediente a probar, de las raciones para el inicio del experimento y de una muestra de los peces del estudio fueron realizados en el
Laboratorio de Análisis Químicos, de la Facultad de Ingeniería Química de la UNAP y en el Laboratorio de Análisis Químico del Centro de Investigación de Quistococha - PEA - IIAP. Estos análisis sirvieron para calcular los tenores de proteína bruta (PB), extracto etéreo ó grasa (EE), extractos no nitrogenados o carbohidratos (ENN), fibra bruta (FB), material mineral ó cenizas (MM) y humedad (HU).

\section{ANALISIS ESTADISTICO}

Los datos fueron procesados en hojas de cálculo de Excel. Para el análisis de varianza (ANOVA), se utilizó el programa estadístico BioStat 2.0 con un nivel de significancia de $95 \%$.

\section{RESULTADOS Y DISCUSIÓN}

PARÁMETROS FÍSICO-QUÍMICOS DELAGUA

Los valores mensuales de los parámetros físicoquímicos del agua dentro y fuera de las jaulas se muestran en la tabla 2. Los valores de temperatura del agua durante los 168 días de cultivo, oscilaron entre 25 a $28{ }^{\circ} \mathrm{C}$ estos valores se encuentran dentro del rango permisible temperatura $\left(20\right.$ a $\left.30{ }^{\circ} \mathrm{C}\right)$ reportado en la literatura por diferentes autores para el cultivo de peces nativos (Díaz \& López, 1993; Guerra et al., 1996; Fonseca \& Storti, 2004). Los registros mensuales de oxígeno disuelto en las jaulas tuvieron un valor promedio de $4 \mathrm{mg} / \mathrm{l}$, valor que está dentro de lo permisible, coincidiendo con Sipaúba (1988) y Guerra et al. (1996) quienes mencionan que para un crecimiento adecuado de los peces, el agua de los estanques debe presentar un tenor de oxígeno disuelto siempre superior a $3 \mathrm{mg} / \mathrm{l}$, valores inferiores a esta concentración provocan una reducción en la conversión alimenticia y un aumento de los efectos perjudiciales resultantes de la degradación de metabolitos.El pH se mantuvo con un valor promedio de 5.5 que está por debajo a los reportados como más adecuados para la piscicultura por Boyd en 1996 y Chuquipiondo \& Galdós en 2005 (entre 7 -8 y $6.2 \pm 0.3$, respectivamente). Guerra et al. (1996) manifiestan que los valores de $\mathrm{pH}$ para el desarrollo normal de la gamitana Colossoma macropomun son de 6.5 a 9. En cuanto a la alcalinidad, este parametro fluctuó entre 2.0 y 4.2 $\mathrm{mg} / 1$, mientras que la dureza fluctuó entre 16.0 a 17.1 $\mathrm{mg} / \mathrm{l}$. Para el cultivo de organismos acuáticos las mejores aguas con respecto a estos dos parámetros son los que tienen valores muy similares. Rodríguez et al. (2001) mencionan que si se presentan valores diferentes, tales como alcalinidad más alta que la dureza, el $\mathrm{pH}$ puede incrementarse a niveles muy altos durante periodos de alta fotosíntesis. 


\section{CRECIMIENTO DE LOS PECES}

Los peces alimentados con la dieta $\mathrm{T} 2$ tuvieron un crecimiento superior (Tabla 3), obteniendo al final del experimento un peso promedio de $60.67 \mathrm{~g}$ si comparamos con las dietas $1, \mathrm{~T} 3$ y $\mathrm{T} 4$ quienes al finalizar el experimento obtuvieron pesos promedio de $56.67 \mathrm{~g}, 51.50 \mathrm{~g}$ y $51.42 \mathrm{~g}$ respectivamente. No se encontró diferencias significativa $(\mathrm{P}>0.05)$, en ninguna de las variables analizadas.

Los resultados del presente trabajo demuestran que los peces alimentados con sacha inchi no tuvieron los resultados esperados. De acuerdo a los resultados la tendencia fue: T2 $>\mathrm{T} 1>\mathrm{T} 3>\mathrm{T} 4$. En el primer mes se puede observar un crecimiento acelerado en el peso de los peces de cada uno de los tratamientos, disminuyendo su ritmo en los siguientes meses, así como también se puede observar en las dietas T1 y T3 sufren un decrecimiento de peso en el último mes (Figura 1).

La curva de crecimiento en longitud del banda negra, se puede observar que los peces de todos los tratamientos tuvieron un crecimiento ascendente, observándose un crecimiento mayor en los peces de la dieta T2, quienes al final del experimento obtuvieron una longitud promedio de $13.01 \mathrm{~cm}$ frente a los de la dietaT1, T3 y T4 que obtuvieron tallas promedio finales de $12.87 \mathrm{~cm}, 12.52 \mathrm{~cm}$ y $12.68 \mathrm{~cm}$ respectivamente. Todas las dietas tuvieron en el primer mes un crecimiento acelerado, disminuyendo su ritmo de crecimiento a partir del segundo mes (Figura 2).

El crecimiento de los peces fue homogéneo durante los 168 días que duró el experimento, sin embargo el nivel de crecimiento de los peces fue pobre y no fue lo esperado, respecto a lo mencionado Santos et al. (2006) Menciona que banda negra, Myleus schomburgkii llega alcanzar los $35 \mathrm{~cm}$. de longitud, aunque no especifica el tiempo en el que llega a tener dicha talla. Por su parte Chuquipiondo \& Galdós (2005) reportaron que la ganancia de peso de los peces durante las 24 semanas que duró el estudio, indicaron que los alevinos de gamitana Colossoma macropomum se desempeñaron pobremente en el cultivo en jaulas, lo que coincide de alguna manera con los resultados obtenidos en el presente estudio. Sin embargo, otros autores tuvieron mejores respuestas en el cultivo en jaulas por ejemplo Padilla (2000) registró pesos de entre 472 y $570 \mathrm{~g}$ de peso en juveniles de gamitana colossoma macropomun cultivados en jaulas por 120 días con 4 dietas diferentes, en base a harina de pescado y ensilado biológico; del mismo modo, Alcántara et al. (2004) reportan ganancias de peso de 343 y $153.5 \mathrm{~g}$ de peso en 180 días respectivamente en un estudio en el que evaluó el cultivo de paco (Piaractus brachypomus) y gamitana (Colossoma macropomum) cultivados en jaulas.
Morias et al. (2001) menciona que una mayor oferta proteica conlleva casi a un mayor incremento de las tasas de crecimiento de los organismos en cultivo. Aunque todas las dietas experimentales tuvieron un alto nivel de proteína proveniente de la harina de sacha inchi $(45,55,65,75 \%)$ por el método de regresión polinomial. Al final del periodo experimental no se encontró diferencia significativa entre los cuatro dietas $(23,25,27$ y $29 \%$ de PB) en el crecimiento de los alevinos de Myleus schomburgkii. Sin embargo, al revisar trabajos que puedan dar soporte a nuestros resultados encontramos trabajos como la de Cantelmo \& De Souza (1986) quienes estudiaron el efecto de raciones balanceadas con cuatro diferentes niveles proteicos $(20,25,30$ y $35 \%)$ en el crecimiento de juveniles de Piaractus brachypomus, no encontrando hasta el final del período experimental, diferencias significativas entre los tratamientos evaluados. asimismo Ruiz \& Vela (2008), mencionan que los tres niveles de inclusión de la torta de sacha inchi y la ración testigo tuvieron el mismo efecto en el crecimiento de la gamitana Colossoma macropomum; Maehana et al. (2004) no hallaron diferencias significativas en la ganancia de peso en ejemplares juveniles del pez disco (Symphysodon sp.), alimentados con dietas entre $35 \%$ y $50 \%$ de proteína. Coincidiendo con el presente estudio, debido a que los cuatro niveles de inclusión del sacha inchi utilizados tuvieron el mismo efecto, no encontrándose diferencia significativa $(\mathrm{P}>0.05)$, al final del experimento.

\section{ÍNDICES ZOOTÉCNICOS}

Los ICAA obtenidos en el presente trabajo, se pudo notar que de todas las dieta utilizadas el T2 fue el mejor, obteniendo al final del experimento un índice de 1.6 en comparación con las dietas T1, T3 y T4, que muestran valores de $1.8,2.2$ y 2.0 respectivamente. En la tabla 4 podemos observar que los peces que obtuvieron mayor ganancia de peso fueron los del Tratamiento 2 (T2) con un peso promedio de $28.40 \mathrm{~g} \mathrm{(} \pm 7.2)$, un incremento de peso de $59.64 \%$, coeficiente de variación de $26.60 \%$, índice hepatosomáticos de $1.65 \%$. Todos los tratamiento presentaron $100 \%$ de supervivencia, resistiendo los peces al manipuleo que se le hizo cada 28 días durante los muestreos. Los ICAA obtenidos en el presente trabajo están dentro de los rangos establecidos, pero con una ganancia de peso bajo. Los peces son conocidos por ser eficientes en la asimilación de ítems alimenticios tanto en condiciones controladas como en su ambiente natural, por lo que consideramos desde el punto de vista acuícola que estos resultados pudieran ser considerados adecuados. Cabe resaltar que tales 
resultados también pueden estar relacionados con el constante y difícil manipuleo en los muestreos biométricos y el porte pequeño de los peces al inicio del experimento, que puede haber causado estrés fisiológico, afectando la asimilación de nutrientes (Tabla 3). Ruiz \& Vela (2008), obtuvieron índices zootécnicos similares a los obtenidos en este estudio con alevinos de gamitana Colossoma macropomum alimentados con raciones conteniendo torta de sacha inchi Plukenetia volubilis, ya que al final del experimento los índices zootécnicos no presentaron diferencia significativa $(\mathrm{P}>0.05)$. El ICAA después de 168 días de cultivo (Tabla 3 ) fueron semejantes a los obtenidos por Bechara et al. (2005) quienes reportaron un ICAA de 1.36, 1.54, 1.73 cuando evaluaron el efecto de los niveles de proteína, calidad de agua y eficiencia de proteína bruta $(25$, $35,45 \%)$ en alevinos de paco, Piaractus brachypomus.

Al final del experimento el tratamiento que obtuvo una mejor tasa de crecimiento específico fue el T2 con un valor de $0.38 \%$, valor muy bajo si lo comparamos con los resultados obtenidos por Eckmann (1987) quien alimentó juveniles de Colossoma macropomum con 6 tipos de raciones conteniendo harina de pescado y harina de sangre de vacuno en varias proporciones. obteniendo promedios de tasas de crecimiento específico entre 1.1 a $2.5 \%$ de peso seco/día, concluyendo que estos eran directamente proporcionales a los niveles de proteína bruta presentes en las raciones, las cuales variaban entre 25 y $37 \%$ como máximo.

El $100 \%$ de tasa de sobrevivencia obtenida en el presente estudio fue mayor a los reportados en la gamitana Colossoma macropomum (alrededor de 90 $\%$, con valores por debajo de $75 \%$ raramente fueron 


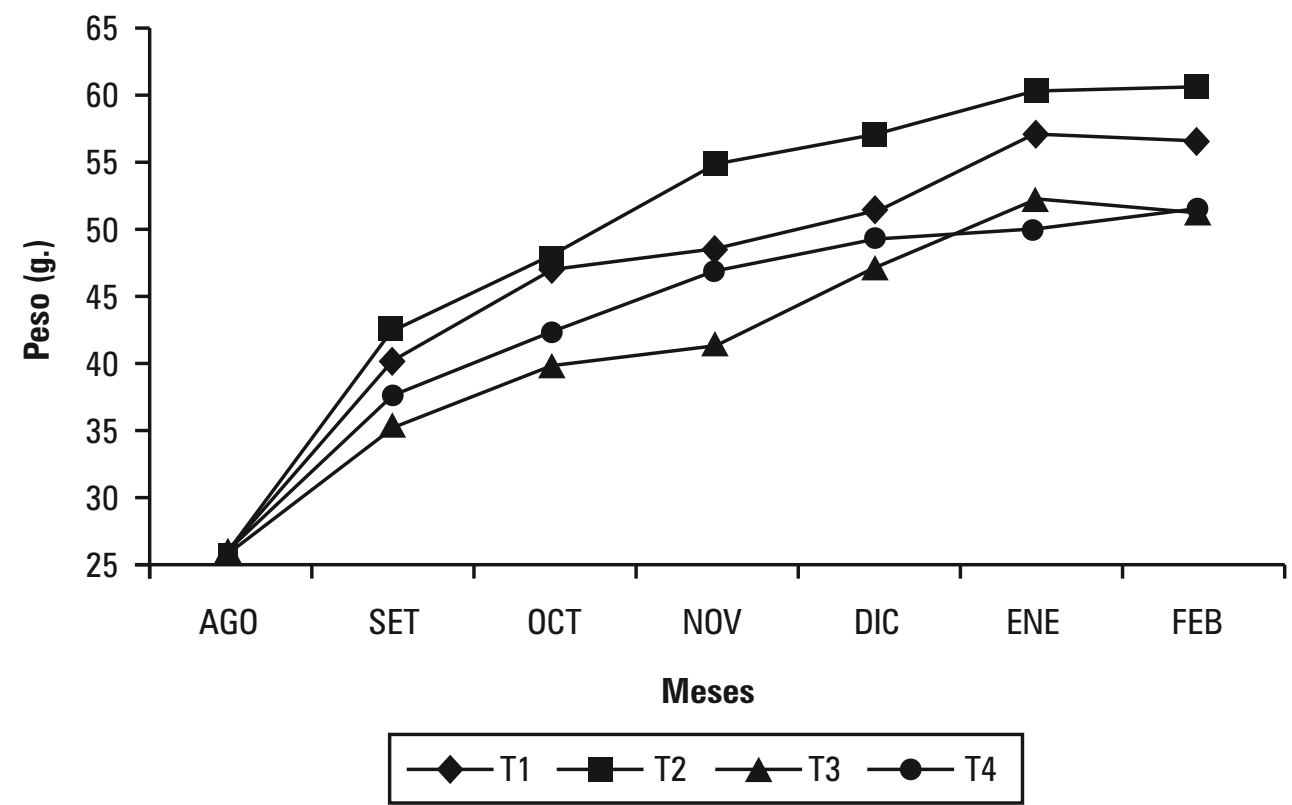

Figura 1. Crecimiento en peso del banda negra, Myleus schomburgkii durante los 168 días de cultivo.
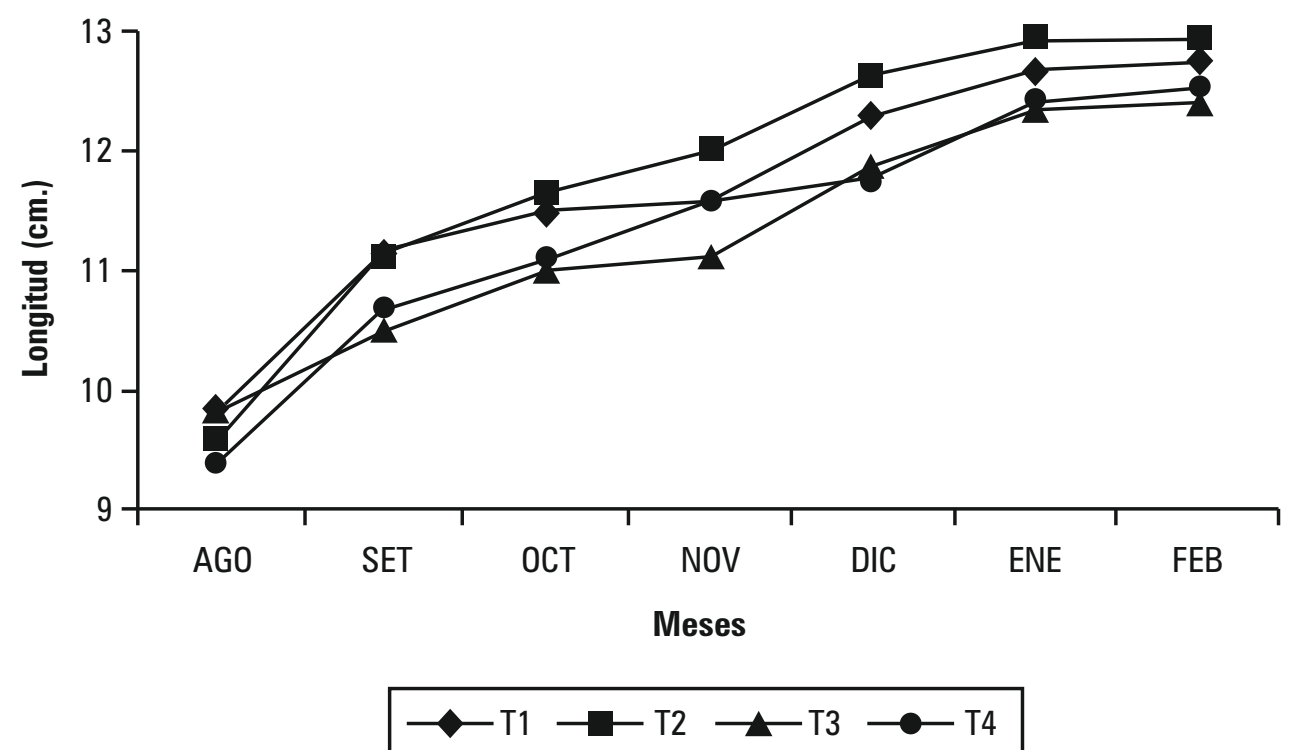

Figura 02.Crecimiento en longitud durante los 168 días de cultivo. Del banda negra, Myleus schomburgkii. 
$\overline{\text { Tabla 1. Composición porcentual (\%) }}$ de cuatros raciones balanceadas utilizados en la alimentación de Myleus schomburgkii raciones experimentales.

\begin{tabular}{lcccc}
\hline \multirow{2}{*}{ Ingredientes } & \multicolumn{4}{c}{ Tratamientos } \\
\cline { 2 - 5 } & T1 & T2 & T3 & T4 \\
\hline Harina de pescado & 10 & 10 & 10 & 10 \\
Harina de sacha inchi & 45 & 55 & 65 & 75 \\
Polvillo de arroz & 20 & 12 & 15 & 10 \\
Harina de maíz & 25 & 23 & 10 & 5 \\
\hline
\end{tabular}

Tabla 2. Calidad del agua (promedio \pm desviación estándar) registrada durante la fase de cultivo de juveniles de banda negra (Myleus schomburgkii ) alimentados con dietas conteniendo harina sacha inchi (Plukenetia volubilis) durante 168 días.

\begin{tabular}{lc}
\hline \multicolumn{1}{c}{ Tratamiento } & $\begin{array}{c}\text { Promedio } \\
\text { Desviación estándar }\end{array}$ \\
\hline Temperatura $\left({ }^{\circ} \mathrm{C}\right)$ & $26.87 \pm 0.97$ \\
Oxígeno $(\mathrm{mg} / \mathrm{l})$ & $4.06 \pm 1.04$ \\
$\mathrm{pH}(\mathrm{upH})$ & $6.01 \pm 0.32$ \\
Alcalinidad $(\mathrm{mg} / \mathrm{l})$ & $3.61 \pm 1.96$ \\
Dureza $(\mathrm{mg} / \mathrm{l})$ & $10.12 \pm 2.06$ \\
Anhídrido carbónico $(\mathrm{mg} / \mathrm{l})$ & $16.93 \pm 0.40$ \\
Amoniaco $(\mathrm{ppm})$ & $0.23 \pm 0.04$ \\
Nitritos $(\mathrm{ppm})$ & $<0.05$ \\
\hline Nitratos $(\mathrm{ppm})$ & $<0.05$ \\
\hline
\end{tabular}


Tabla 3. Índices de crecimiento (promedios \pm error estándar de la media) obtenidos en el cultivo de juveniles de Banda negra (Myleus schomburgkii) alimentados con dietas conteniendo tres niveles de inclusión harina sacha inchi (Plukenetia volubilis) durante 168 días.

\begin{tabular}{lcccc}
\hline \multirow{2}{*}{ Variable } & \multicolumn{4}{c}{ Tratamientos } \\
\cline { 2 - 5 } & T1 & T2 & T3 & T4 \\
\hline PCl (g) & $26.17 \pm 4.32$ & $25.50 \pm 4.25$ & $26.25 \pm 4.37$ & $25.92 \pm 4.32$ \\
\hline PCF (g) & $56.17 \pm 12.84$ & $60.67 \pm 10.11$ & $51.50 \pm 8.58$ & $51.42 \pm 8.57$ \\
GP (g) & $24.01 \pm 4.10$ & $28.4 \pm 4.73$ & $18.49 \pm 3.0$ & $20.39 \pm 3.39$ \\
GPD (g) & $0.80 \pm 0.13$ & $0.95 \pm 0.15$ & $0.61 \pm 0.10$ & $0.68 \pm 0.11$ \\
BG (kg) & $0.36 \pm 0.06$ & $0.42 \pm 0.07$ & $0.32 \pm 0.05$ & $0.36 \pm 0.06$ \\
\hline
\end{tabular}

Leyenda: Peso corporal inicial: PCl, Peso corporal final: PCF, Ganancia de peso: GP,

Ganancia de peso diario: GPD, Biomasa ganada: $B G$.

Tabla 4. Índices zootécnicos (promedios \pm error estándar de la media) obtenidos en el cultivo de juveniles de banda negra (Myleus schomburgkii) alimentados con dietas conteniendo cuatro niveles de inclusión de harina de sacha inchi(Plukenetia volubilis) durante 168 días

\begin{tabular}{|c|c|c|c|c|}
\hline \multirow[b]{2}{*}{ Variable } & \multicolumn{4}{|c|}{ Tratamientos } \\
\hline & T1 & $\mathrm{T} 2$ & T3 & T4 \\
\hline ICAA & $1.8 \pm 0.13$ & $1.6 \pm 0.1$ & $2.2 \pm 0.4$ & $2.0 \pm 0.1$ \\
\hline GP & $24.1 \pm 6.38$ & $28.4 \pm 7.2$ & $18.49 \pm 6.7$ & $20.39 \pm 5.1$ \\
\hline IP & $53.2 \pm 0.40$ & $59.6 \pm 6.7$ & $44.42 \pm 10.8$ & $48.14 \pm 0.4$ \\
\hline TCE & $0.33 \pm 0.60$ & $0.38 \pm 0.7$ & $0.25 \pm 0.4$ & $0.29 \pm 0.5$ \\
\hline S & 100 & 100 & 100 & 100 \\
\hline IHS & 1.7 & 1.6 & 1.9 & 1.9 \\
\hline
\end{tabular}

Leyenda: ICAA = Índice de conversión alimenticia aparente; GP = Ganancia de Peso; $I P=$ Incremento de peso; TCE = Tasa de crecimiento específico; $S=$ Supervivencia e IHS = Índice hepatosomático 
Chota, W.; Vásquez, G.; Del Castillo, D.; Renno, J,F.; García-Dávila, C. 2008. Diversidad Genética en Poblaciones Naturales de Sacha Inchi Plukenetia volubilis L. (Euphorbiaceae) en el Departamento de San Martín (Perú). Folia Amazónia. Vol. 17 No 1-2, p. 83 -90.

Cowey, C.B. 1979. Exigências de proteínas e aminoácidos pelos peixes. In: Fundamentos de Nutrición. N. Castagnolli (Edit.). UNESP. Campus de Jaboticabal. Faculdade de Ciências Agrarias e Veterinarias. 31-41 p.

Boyd, C. 1996. Manejo de suelos y de la calidad de agua en la Acuicultura de piscinas. Asociación Americana de Soya (ASA). Caracas, Venezuela. 62pp.

Cantelmo, A.; Souza, J. A. 1986. Influencia da alimentação em diferentes níveis proteicos para o desenvolvimento inicial do pacu Colossoma mitrei. In: Sintese de trabalhos realizados com espécies do gênero Colossoma. Projeto Aquicultura. CPTA. Pirassununga.

Chagas, E.C.; Val, A.L. 2003. Efeito da vitamina C no ganho de peso e em parâmetros hematologicos de tambaqui. Pesq. Agropec. Bras. Brasilia. V. 38, n. 3, p. 397-402.

Chuquipiondo, J.M.L.; Galdós, R.A.P. 2005. Influencia de La harina de plátano, Musa paradisíaca L. Em el crecimiento de alevinos de gamitana Colossoma macropomum (Cuvier 1818).

Díaz, F.; López R. 1993. EL cultivo de la "Cachama blanca" (Piaractus brachypomus) y de la "cachama negra" (Colossoma macropomum). Fundamentos de Acuicultura Continental. Ministerio de Agricultura, Instituto Nacional de Pesca y Acuicultura (INPA). Bogotá, Colombia. p. 207-219.

Eckmann, R. 1987. Growth and body composition of juvenile Colossoma macropomum CUVIER 1818 (Characidae) feeding artificial diets, Aquaculture; 64: 293-303.

Guerra, F.H.; Alcántara, B.F.; Campos, B.L. 1996. Piscicultura Amazónica con Especies Nativas. Tratado de Cooperación Amazónica (T.C.A) Secretaria Pro - Tempore. Mirigraf. S.R.L. Lima -Perú.138 pp.

Guerra,H.; Saldaña, G. 2002. Cultivando peces amazónicos. IIAP/IRG/BIOFOR/MP. San Martín-Perú. 200 pp.

Manco, C.E. 2005. INIEA - Sudirgeb - EEA. "El Porvenir, Cultivo de Sacha inchi.San Martín Perú.p.2-3.

Morias, S.; Bell, J.G.; Robertson, D.A.; Roy, W.J.; Morris, P.C. 2001. Protein/lipid ratios in extruded diets for atlantic cod (Gadus morhua L.): effects on growth, feed utilization, muscle c o m p o s i t i o n a n d 1 i v e r histology.Aquaculture, 203:101-119.

Padilla, P. 2000. Efecto del contenido proteico y energético de dietas en el crecimiento de alevinos de gamitana (Colossoma macropomum). Folia Amazónica. Vol. 10 (1-2).

Rodríguez, H; Victoria, P; Carrillo, M. 2001. Fundamentos de acuicultura continental. INPA/MADR. Bogotá-Colombia. 423 pp.

Ruiz, J.A.; Vela, E.M. 2008. Utilización de la torta de sacha inchi, Plukenetia volubilis (Euphorbiaceae) en raciones alimenticias para alevinos de gamitana, Colossoma macropomum (Serrasalmidae) criados en jaulas flotantes. Tesis de pre-grado. Universidad Nacional de la Amazonia Peruana. Iquitos-Perú. 74 p.

Sánchez, R.H.; Padilla, P.P.; Vásquez, N.B. 2005. Comportamiento Reproductivo y Crecimiento de Chaetobranchus semifasciatus "bujurqui tucunare" en Ambientes Controlados. Sesión Especial de Investigación en Acuicultura. Taller Internacional para el Desarrollo de la Acuicultura Continental Amazónica. Iquitos Perú. 6p.

Saint-Paul, U. 1984. Ecological and physiological investigations on Colossoma macropomum, new specie for fish culture in Amazons.Mems. Asoc. Latinoamérica. Acuicult., 5(3): 501-518.

Santos, G.; Ferreira, E.; Zuanon, J. 2006. Peixes Comerciais do Manaus. Edições Ibama. Manaus: Ibama/AM, Pro Várzea, p. 40-43.

Sipaúba, L. 1988. Limnología Aplicada a Aqüicultura. Universidade Estadual Paulista NESR. BolTecn ${ }^{\circ}$ Centro de Aqüicultura. 71p.

Tibaldi, Y.; Tulli, F.; Ballestrazzi, R.; Lanardi, D., 1991. Effect of dietary protein/metabolizable energy ratio and body size on the performance of juvenile sea bass.Zootech. Nutr. Anim., vol. 17, $\mathrm{n}^{0} .5,313-320 \mathrm{p}$. 
FOLIA

Plukenetia volubilis (EUPHORBIACEAE) EN EL CRECIMIENTO DE ALEVINOS DE BANDA NEGRA

Amazónica $\quad$ Myleus schomburgkii (PISCES, SERRASALMIDAE) CRIADOS EN CAUTIVERIO 